\title{
QCD at finite temperature and density on the lattice
}

Maria Paola Lombardo

INFN, Laboratori Nazionali di Frascati, Italy

\begin{abstract}
In the first lecture we briefly summarize the basics of field theory thermodynamics and critical phenomena. We then introduce the lattice gauge field theory approach to QCD at finite temperature and density, which is a non-perturbative scheme allowing first principle calculations using the QCD Lagrangian as a sole input. Some of the general concepts and idea introduced at the beginning are demonstrated by use of simple effective models of QCD. The second lecture is devoted to applications. We emphasize that current methods suffice to study the main phenomena at RHIC and LHC energies, and we discuss the ongoing theoretical efforts devoted to the solution of the sign problem which hampers the simulations of cold and dense matter. We conclude with short overview of the status of the field as of Summer 2008.
\end{abstract}

\section{References}

1. The Proceedings of Quark Matter, the International Conference on Ultrarelativistic Heavy Ion Collisions, usually include reviews on recent lattice results; the proceedings of The International Symposium on Lattice Field Theory usually include review on more specific aaspects of lattice thermodynamics.

2. M. P. Lombardo, Finite temperature field theory and phase transitions, arXiv:hep-ph/0103141. Lectures given at ICTP Summer School on Astroparticle Physics and Cosmology, Miramare, Trieste, Italy, 12 Jun - 7 Jul 2000. Published in *Trieste 2000, Astroparticle and Cosmology*

3. F. Karsch, Lattice $Q C D$ at high temperature and density, Lect. Notes Phys. 583, 209 (2002) [arXiv:heplat/0106019].

4. J. B. Kogut and M. A. Stephanov, The phases of quantum chromodynamics: From confinement to extreme Camb. Monogr. Part. Phys. Nucl. Phys. Cosmol. 21 (2004) 1 .

This is an Open Access article distributed under the terms of the Creative Commons Attribution-Noncommercial License 3.0, which permits unrestricted use, distribution, and reproduction in any noncommercial medium, provided the original work is properly cited. 


\section{Helmholtz International Summer School Dense Matter in Heavy Ion Collisions and Astrophysics JINR Dubna Russia July 14-26 2008 $\underline{Q C D}$ at finite temperature and density on the lattice} M.P. Lombardo, INF'N Laboratori Nazionali di Frascati

Thermodynamics Basics

Thermodynamical Eusembles

- Microcanonical: E, N, V fixed

- Canonical: T, N, V fixed

- Grancanonical : T, $\mu$, V fixed

In a GrandCanonical Ensemble define:

$$
\begin{aligned}
\hat{\rho} & =e^{-(H-\mu \hat{N}) / T} \\
\mathcal{Z} & =\operatorname{Tr} \hat{\rho}
\end{aligned}
$$


$\rho$ e $\mathcal{Z}$ determine the system's state:

$$
\begin{aligned}
&<O>=\operatorname{Tr} O \hat{\rho} / \mathcal{Z} \\
& P=T \frac{\partial \ln \mathcal{Z}}{\partial V} \\
& N=T \frac{\partial \ln \mathcal{Z}}{\partial \mu} \\
& S=\frac{\partial T \ln \mathcal{Z}}{\partial T} \\
& E=-P V+T S+\mu N
\end{aligned}
$$

Bosons and fermions

- 1 bosonic degree of freedum

$$
\begin{gathered}
\hat{H}=1 / 2 \omega a^{\dagger} a+1 / 2 \omega=\omega(\hat{N}+1 / 2) \\
\mathcal{Z}=\operatorname{Tr} e^{-\beta(H-\mu \hat{N})}=\operatorname{Tr} e^{-\beta(\omega-\mu) \hat{N})} \\
=\sum_{0}^{\infty} \operatorname{Tr} e^{-\beta(\omega-\mu) n}=\left(1-e^{-\beta(\omega-\mu) n}\right)^{-1} \\
N=\left(e^{\beta(\omega-\mu)}-1\right)^{-1}
\end{gathered}
$$

- 1 fermionic degree of freedom

$$
\begin{gathered}
\hat{H}=1 / 2 \omega a^{\dagger} a-1 / 2 \omega=\omega(\hat{N}-1 / 2) \\
Z=\operatorname{Tr}^{-\beta(H-\mu \hat{N})}=\operatorname{Tr} e^{-\beta(\omega-\mu) \hat{N})} \\
=\sum_{0}^{1} \operatorname{Tr} e^{-\beta(\omega-\mu) n} \\
N=\left(e^{\beta(\omega-\mu)}+1\right)^{-1}
\end{gathered}
$$


Example I : Relativistic free fermionic gas

Non interacting particles: $\mathcal{Z}$

$$
\begin{gathered}
\mathcal{Z}=\prod \mathcal{Z}^{\prime} \\
\ln \mathcal{Z}=d^{3} x \int d^{3} p\left(\ln \left(e^{-\beta(\omega-\mu)}+1\right)+\ln \left(e^{-\beta(w+\mu)}+1\right)\right)
\end{gathered}
$$

Note! Relativistic particles!

- Fermions $(+\mu)$ and antifermions $(-\mu)$.

- $\omega=\sqrt{\left(p^{2}+m^{2}\right)}$.

- Relativistic chemical potential

$\mathcal{Z}$ can be exactly computed in the chiral limit $m=0$ :

$$
\begin{aligned}
T \ln Z & =V \mu^{4} /\left(12 \pi^{2}\right)+V \mu^{2} T^{2} / 6+7 V \pi^{2} T^{4} / 180 \\
n & =4 V \mu^{3} / 12 \pi^{2}+2 V \mu T^{2} / 6
\end{aligned}
$$

Ideally, this should be the state of the quarks at very high temperatures!

APPROACH TO FREE FIELD : ANALYTIC RESULTS VS. LATTICE DATA Based on A. Vuorinen, 2004
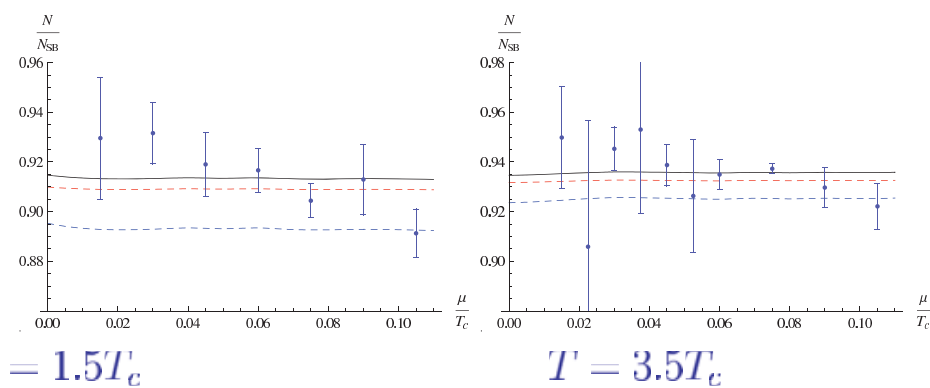

$T=1.5 T_{c}$

$T=3.5 T_{c}$

D'Elia, Di Renzo, MpL, Vuorinen, in progress 
More observables : Response Functions Susceptibilities

$$
\chi_{j_{u}, j_{d}}(T)=\left.\frac{\partial^{\left(j_{u}+j_{d}\right)} p\left(T, \mu_{u}, \mu_{d}\right)}{\partial \mu_{u}^{j_{u}} \partial \mu_{d}^{j_{d}}}\right|_{\mu_{u}=\mu_{d}=0} .
$$

Test for fluctuations.

Taylor coefficients of the excess pressure:

$$
\begin{gathered}
\Delta p\left(T, \mu_{u}, \mu_{d}\right) \equiv p\left(T, \mu_{u}, \mu_{d}\right)-p\left(T, \mu_{u}=0, \mu_{d}=0\right) \\
\Delta p\left(T, \mu_{u}, \mu_{d}\right)=\sum_{j_{u}, j_{d}} \chi_{j_{u}, j_{d}}(T) \frac{\mu_{u}^{j_{u}}}{j_{u} !} \frac{\mu_{d}^{j_{d}}}{j_{d} !},
\end{gathered}
$$

containing information about baryon density effects in the EoS.

Susceptibilities Towards Free Field

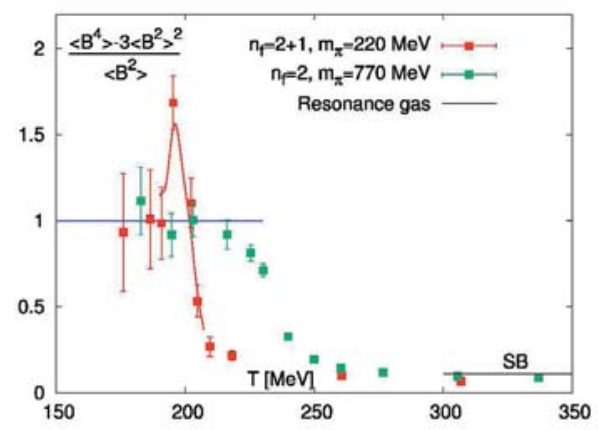

RBC-Bielefeld Collaboration, 2008 
Example II : Interacting Fermions

3 d Gross Neveu Model

$$
L=\bar{\psi}(\partial+m) \psi-g^{2} / N_{f}\left[(\bar{\psi} \psi)^{2}-\left(\bar{\psi} \gamma_{5} \psi\right)^{2}\right]
$$

Global Chiral Invariance

$$
\begin{aligned}
& \psi_{i} \rightarrow e^{i \alpha \gamma_{5}} \psi_{i} \\
& \bar{\psi}_{i} \rightarrow \bar{\psi}_{i} e^{i \alpha \gamma_{5}}
\end{aligned}
$$

Basic Properties

- A $T=\mu=0$ and $g$ 'large', spontaneous symmetry breaking, Goldstone mechanism.

- Rich particle spectrum

- Amenable to a lattice study at $T, \mu \neq 0$ !!!!

Phase diagram of the 3d Gross Neveu model

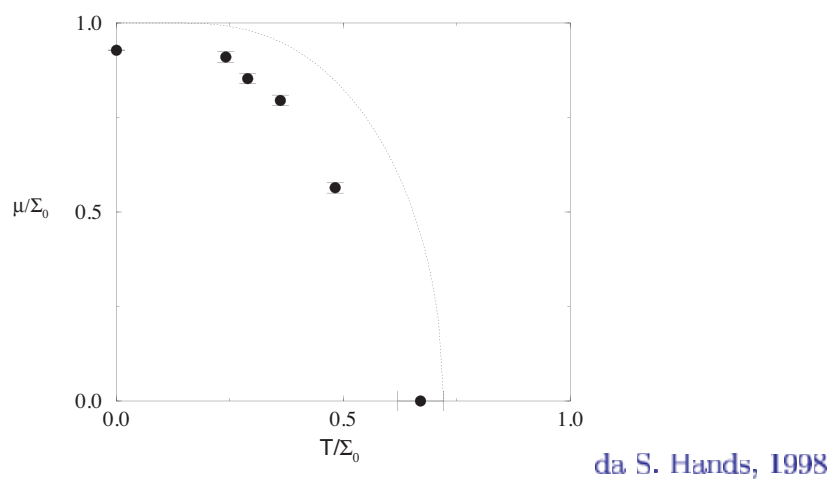

Mean Field Solution vs Exact Lattice Results 
- Grand Canonical Formalism at finite T, $\mu$

- Basic Observables : number density, susceptibilities

- Free Fermions : Exact Solution

- Simple model with interacting fermiuns : mean field solution

- Phase Diagram at nonzero T and $\mu$ of a purely fermionic model

- Simple calculations : can reproduce limiting behaviour and give generic information; in general inaccurate

$$
\begin{gathered}
\text { Lattice } Q C D \\
= \\
\text { first principles calculations from the } \\
Q C D \text { Lagrangian } \\
\mathcal{L}=\mathcal{L}_{Y M}+\bar{\psi}\left(i \gamma_{\mu} D_{\mu}+m+\mu \gamma_{0}\right) \psi
\end{gathered}
$$

A vaste phase space to be explored:

Real baryon chemical potential, temperature, isospin chemical potential. And also: Imaginary chemical potentiul, number of color and number of flavor, bare masses

to address phenumenological issues as well as to study more theoretical questions 
Phenomenological challenges:

ab initio study of the QCD phase diagram

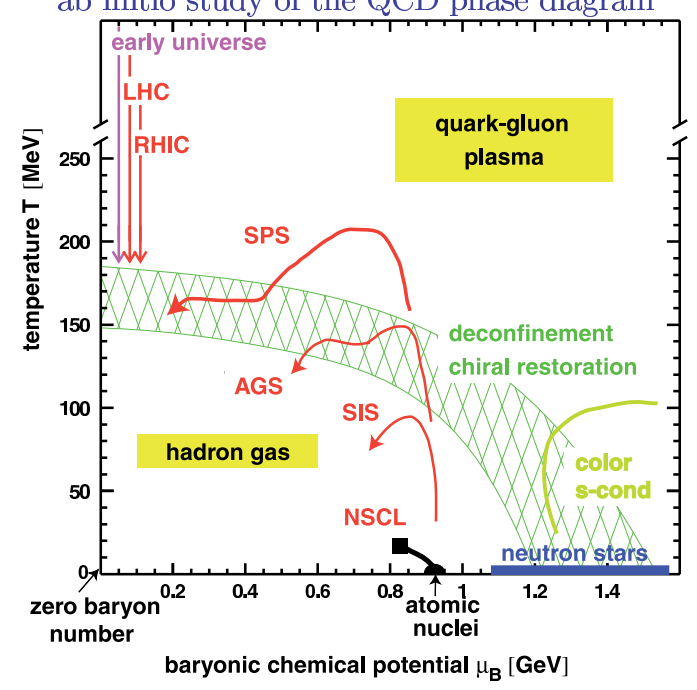

Theoretical questions can (or, rather, should ?) be addressed in a larger phase space:

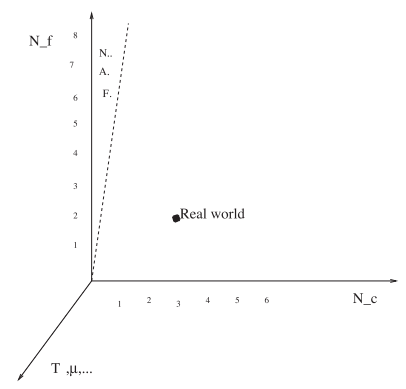


- High $T$

- Chiral symmetry pattern : order disorder, light baryons

- Deconfinement/screening: string breaking via recombination with light pairs

- Instanton molecules

- High $\mu$

- Chiral symmetry pattern : instability at the Fermi surface

- Deconfinement/screening : string breaking via recombination with real particles

- Instanton chains

Differences at high $\mathrm{T}$ and high $\mu$ in the gauge dynamics provide futher motivation to study nonzero $\mu$ on a lattice.

\section{Lattice QCD at Finite Temperature and Density}

Lecture I

I Formulation

II Calculational Schemes

II.1 E'ffective Fermionic Models - Analytic approaches

II.2 E'ffective Gluonec Models - Numeracal approaches

III QCD at Finite Baryon Density: Methods

III.1 Derivatives

III.2 Reweighting

III.3 Expanded Reweighting

III.4 Imaginary Chernical Potential

IV Results - Discussion : Tomortow's lecture 


\section{Formulation}

GranCanonical Formalism and Path Intergral

* Chemical Potential for Conserved Charge $\hat{N}$

$$
\begin{aligned}
& \tilde{\rho}=\mathrm{e}^{-(H-\mu \hat{N}) / T} \\
& \check{z}=\operatorname{Tr} \hat{\rho}=\int d \phi d \psi \mathrm{e}^{-S(\phi, \psi)}
\end{aligned}
$$

* Temperature: Reciprocal of Imaginary Time

$$
S(\phi, \psi)-\int_{0}^{1 / T} d t \int d^{d} x \mathcal{L}(\phi, \psi)
$$

weth boundary condetions for fermions and bosons

$$
\begin{aligned}
& \phi(t=U, \vec{x})=\phi(t=1 / T, \vec{x}) \\
& \psi(t-U, \vec{x})--\psi(t-1 / T, \vec{x})
\end{aligned}
$$

$\star \mathcal{Z}=$ partition function of a statistical system in $d+1$ dimension, where $T$ is the reciprocal of the imaginary time.

* Thermodynamics and spectrum properties are treated on the same footing.

Lattice QCD at $\mathbf{T}, \mu \neq 0$

* Temperature: as in the continuum $T=1 / N_{t} * a$

$\star$ Density

In the continuum: $L(\mu)=L_{0}+\mu J_{0} \quad J_{0}=\bar{\psi} \gamma_{0} \psi \rightarrow N-\bar{N}=\int J_{0}$

On the lattice:

$L(\mu)=\bar{\psi}_{x} \gamma_{0} e^{\mu a} \psi_{x+0 \hat{0}}-\bar{\psi}_{x+\hat{0}} \gamma_{0} e^{-\mu a} \psi_{x}$

$J_{0}=-\partial_{\mu} L=\bar{\psi}_{x} \gamma_{0} e^{\mu a} \psi_{x+\hat{0}}+\bar{\psi}_{x+\hat{0}} \gamma_{0} e^{-\mu a} \psi_{x}$

Time Forward propagation enhanced by $e^{\mu a}$

Time Backward propagation discouraged by $e^{-\mu a}$

Particles-antiparticles asymmetry! 
More on The Lattice (digression)

Path integral is a regulated on a four dimensional lattice

- Gauge fields: link variables $U_{\mu}(x)$ for parallel trasport of field A from $x$ to $x+\hat{A} a$ $\underset{U_{\mu}(x)}{\longrightarrow} x+\hat{\mu} a$

$$
U_{x, \mu}-\mathrm{P} \exp \left(i g \int_{x}^{x+\hat{\mu} a} d x^{\mu} A_{\mu}(x)\right)
$$

- Guuge invarzants and Yang Mill Action:

$$
\begin{aligned}
W_{n, \mu \nu}^{(1,1)} & =1-\frac{1}{3} \operatorname{Re}{ }_{n, \mu \nu} \\
& =\operatorname{Re} \operatorname{Tr} U_{n, \mu} U_{n+\hat{\mu}, \nu} U_{n+\mathcal{D}, \mu}^{\dagger} U_{r, \nu}^{\dagger} \\
& =\frac{g^{2} a^{4}}{2} F_{\mu \nu}^{a} F_{\mu \nu}^{a}+\mathcal{O}\left(a^{6}\right)
\end{aligned}
$$

- Lattice Yang Mill Action

$$
\beta S_{G}=\beta \sum_{\substack{n \\ 0 \leq \mu<\nu \leq 3}} W_{n, \mu \nu}^{(1,1)} \rightarrow \int d^{4} x \mathcal{L}_{Y M}+\mathcal{O}\left(a^{2}\right)
$$

$\beta=6 / g(a)^{2}$.

- Lattice fermions

Simply:

$\psi(x) \rightarrow \psi(n) !$

$\left.\partial_{\mu} \psi_{f}(\boldsymbol{x})-\left(\psi(\boldsymbol{n}+\hat{A})-\psi_{(} \boldsymbol{n}-\hat{A}\right)\right) / 2 a$,

[doubling problem and chtral symuetry: staggered fermoons, Wilson fermons, chiral fermions]

$\mu \bar{\psi} \gamma_{\cup} \psi$ on the lattice

Naive descretisation:

$$
\begin{aligned}
\phi_{L A T T}\left(n_{1}, n_{2}, n_{3}, n_{4}\right) & -\phi\left(n_{1} a, n_{2} a, n_{3} a, n_{4} a\right) \\
\Delta_{\mu} \phi_{L A T T}\left(n_{1}, n_{2}, n_{3}, n_{4}\right) & = \\
\left(\phi_{(} a,\left(n_{\mu}+1\right) a, n_{3} a, n_{4} a\right) & -\phi\left(n_{1} a,\left(n_{\mu} a, n_{3} a, n_{4} a\right)\right) / 2 a
\end{aligned}
$$

Problems with free fermions: the internal energy $\in$ diverges on the continuum limit $a \rightarrow \square$

$$
\begin{gathered}
L=\bar{\psi}_{\boldsymbol{x}} \gamma_{\mu} \psi_{x+\mu a}+m \bar{\psi}_{x} \psi_{\boldsymbol{x}}+\mu \bar{\psi}_{\boldsymbol{x}} \gamma_{U} \psi_{\boldsymbol{x}} \\
\in \propto \times \frac{\mu^{2}}{a^{2}} \rightarrow_{a \rightarrow U} \infty
\end{gathered}
$$

Elegant solution : $\mu$ is an external field in the Uth direction

$$
\bar{\psi} \gamma_{\mu} A_{\mu} \psi \leftarrow \rightarrow i \mu \bar{\psi} \gamma_{0} \psi
$$

- External fields live on lattece link. (cfr. electrodynamics: $\left.A \rightarrow \theta-e^{(i} A\right)$ )

- $L(\mu)=\bar{\psi}_{x} \gamma_{U} e^{\mu a} \psi_{x+0}-\bar{\psi}_{x+0} \gamma_{\cup} e^{-\mu a} \psi_{x}$ 
EPJ Web of Conferences

- Simple intepretation

- Time Forward propagation enhanced by $e^{\mu a}$

- Time Backward pmpagation discouraged by e- $-\mu a$

Purticles-antiparticle asymmetry!

- $\lim _{a \rightarrow \square} J_{0}=-\partial_{\mu} L=\bar{\psi}_{x} \gamma_{U} e^{\mu a} \psi_{x+U ̂}+\bar{\psi}_{x+0 ̂} \gamma_{U} e^{-\mu a} \psi_{x}=\mu \bar{\psi} \gamma_{0} \psi$

Via an unstary transformation for the field

$$
L(\mu)=L(U)
$$

+ boundary conditions

Explecit dependence on fugacity

Lattice QCD Thermodynamics at a Glance

$$
\begin{aligned}
& \mathcal{L}_{Q C D}-6 / g^{2} \operatorname{Tr} U_{n, \mu} U_{n+\hat{\mu}, \nu} U_{n+\hat{\nu}, \mu}^{\dagger} U_{n, \nu}^{\dagger} \\
& +\sum_{i=1}^{3}\left(\bar{\psi}_{\boldsymbol{x}} \gamma_{i} U_{i}(\boldsymbol{x}) \psi_{\boldsymbol{x}+\hat{i}}-\bar{\psi}_{\boldsymbol{x}+\hat{i}} \gamma_{\boldsymbol{i}} U_{i}^{\dagger}(\boldsymbol{x}) \psi_{\boldsymbol{x}}\right) \\
& +\bar{\psi}_{x} \gamma_{0} e^{\mu} U_{0}(x) \psi_{x+0}-\bar{\psi}_{x+0} \gamma_{0} e^{-\mu} U_{0}^{\dagger}(x) \psi_{x} \\
& +m \bar{\psi} \psi
\end{aligned}
$$

Imaginary time

and

Inverse

Temperature

d-dimensional space 
II Computational Schemes

$$
\mathcal{Z}-\int_{\mathcal{L}_{Q C D}} d \phi d \psi e^{-S(\phi, \psi)} ;(\phi, \psi)-\int_{0}^{1 / T} d t \int d^{d} d^{d} \mathcal{L}(\phi, \psi)
$$

Two optrons:

1. Integrate out gluons first:

$$
\mathcal{Z}(T, \mu, \bar{\psi}, \psi, U) \simeq \mathcal{Z}(T, \mu, \bar{\psi}, \psi) \rightarrow
$$

effective approximate fermion models

2. Integrate out fermeons exactly as $S$ is bilmear in $\psi, \bar{\psi}$ $S=S_{Y M}(U)+\bar{\psi} M(U) \psi$

$$
\mathcal{Z}(T, \mu, U)-\int d U e^{-\left(S_{Y M}(U)-\log (\operatorname{det} M)\right)}
$$

$$
\mathcal{Z}(T, \mu, U)=\int d U e^{-\left(S_{Y M}(U)-\log (\operatorname{det} M)\right)}
$$

starting point for numerical calculations 
EPJ Web of Conferences

I1, 1 Effective Fermionic Models on the Lattice

Lattice Strong Coupling Calculations:

Starting point : Yang Mill Action decouples

at $g=\infty \rightarrow \int d U$ exact

Work on two color QCD by Y. Nishida, K. Fukushima, and T. Hatsuda

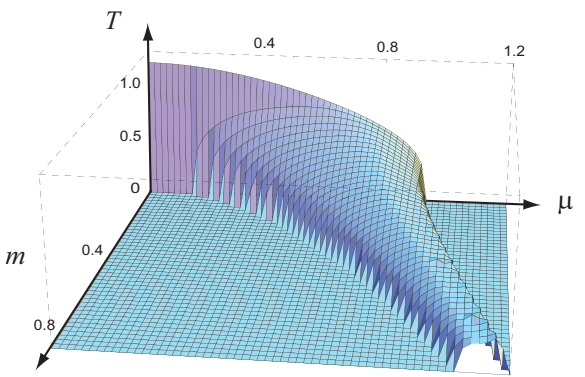

Initial studies by F. Karsch, U. Wollf, and others.

Work from the 80's on three color:

B. Petersson and collaborators, P. Damgaard, F. Karsch and many others.

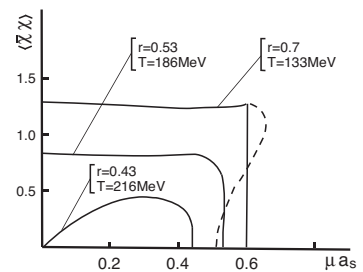

$\mathcal{L}=G \frac{1}{8 N_{c}^{2}}\left[\left(\bar{\psi} \tau^{-} \psi\right)^{2}+\left(\bar{\psi} \tau^{-} \gamma_{5} \psi\right)^{2}\right]$

$C S C / C F L$ Phase from Strong Coupling? 


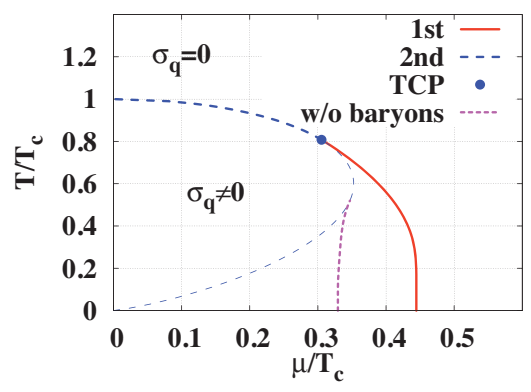

The phase diagram of QCD at strong coupling Kawumoto et al, 2005.

$$
\begin{gathered}
\mathcal{Z}(T, \mu, U)=\int d U e^{-\left(S_{Y M}(U)-\log (\operatorname{det} M)\right)} \\
M^{\dagger}\left(\mu_{B}\right)=-M\left(-\mu_{B}\right)
\end{gathered}
$$

$\operatorname{det} M>0 \rightarrow$ Importance Sampling

- $\mu=0 \rightarrow \operatorname{det} M$ is real

Particles-antiparticles symmetry

- Imaginary $\mu \neq 0 \rightarrow \operatorname{det} M$ is real (Real) Particles-antiparticles symmetry

- Real $\mu \neq 0$ Particles-antiparticles asymmetry $\rightarrow \operatorname{det} M$ is complex in QCD

- QCD with a real baryon chemical potential: use information from the accessible region 
EPJ Web of Conferences

$\operatorname{Re} \mu=0, \operatorname{Im} \mu \neq 0$

\section{QCD AT FINITE BARYON DENSITY-METHODS}

QCD and a Complex $\mu_{B}$

A map: complex $\mu \rightarrow$ complex $\mu^{2}$.

$\mathcal{Z}\left(\mu^{2}\right)$ is real valued for real $\mu^{2}$

Analogy with statistical models in external fields

The Phase Diagram in the T, $\mu_{B}^{2}$ Plane Region accessible to simulations: $\mu^{2}$ real $\leq 0$.

- $\mu=0$ Derivatives, Reweighting, Expanded reweighting

- $\mu^{2} \leq 0$ Imaginary chemical potential 


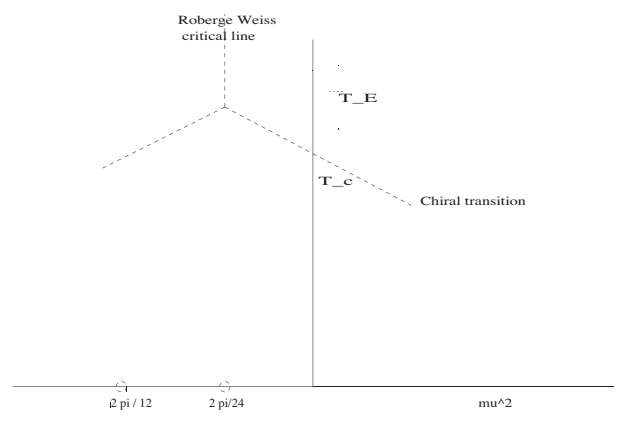

\section{The Roberge and Weiss analysis}

$$
\mathcal{Z}(\nu)=\operatorname{Tr} e^{-\beta H+i \beta \nu N}=e^{-\beta H+i \theta N}
$$

1. $\mathcal{Z}(\theta)$ has a periodicity $2 \pi$ anyway.

2. If only color singlet are allowed, then $N=0 \bmod \left(N_{c}\right)$ and periodicity becomes $2 \pi / N_{C}$

However (Roberge Weiss (1986))

$\mathcal{Z}(\theta)$ has always period $2 \pi / N_{c}$

The imaginary chemical potential changes the preferred vacuum for the Polyakov loop from $\phi_{P}=0$ to one of its $Z_{3}$ images

The strong coupling analysis shows that periodicity is smooth at low temperature, and p.t. theory suggests that it is sharp at high $T$ 
III. 1 Derivatives at $\mu-U . U$
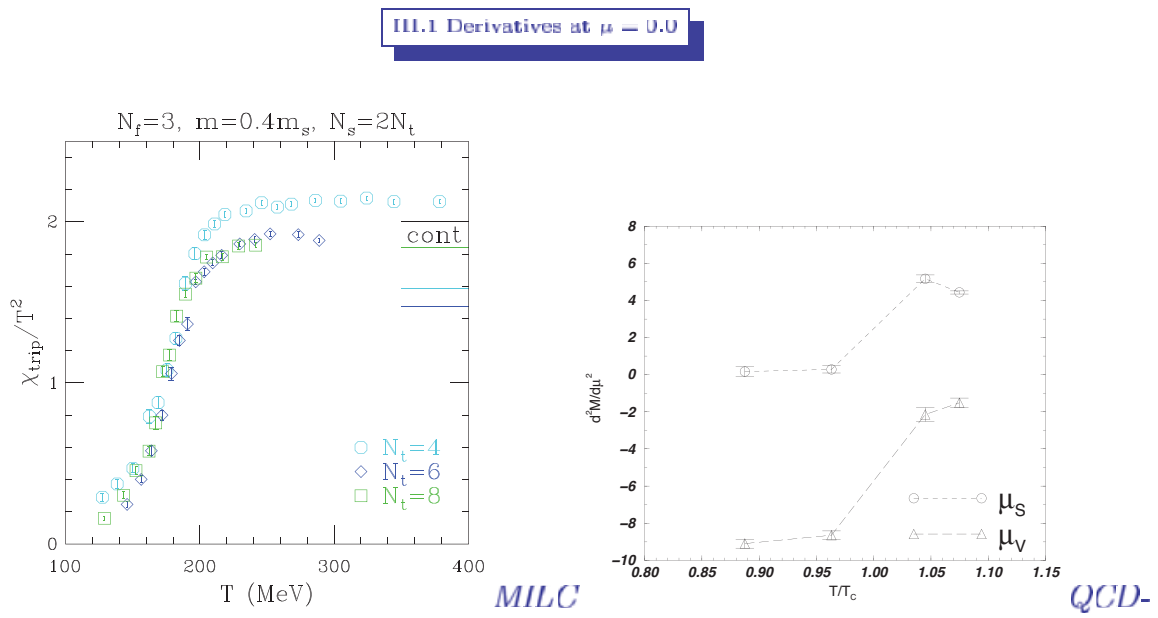

TARO

Ian Barbour's proposal, or

The Glasgow method:

$\mathcal{Z}(\mu)$ can be computed using simulations at $\mu=0$ :

$$
\begin{gathered}
\mathcal{Z}=\left\langle\frac{|M(\mu)|}{|M(\mu=0)|}\right\rangle_{\mu=0} \\
\mathcal{Z}=\frac{\int[d U]\left[d U^{\dagger}\right]|M(\mu)| e^{-S_{g}\left[U, U^{\dagger}\right]}}{\int[d U]\left[d U^{\dagger}\right]|M(\mu=0)| e^{-S_{g}\left[U, U^{\dagger}\right]}}
\end{gathered}
$$

Neeeds overlap between

simulation ensemble at $\mu=0$

target ensemble at $\mu \neq 0$

At $T=0$ the Glasgow procedure fuils because of a poor overlap. 


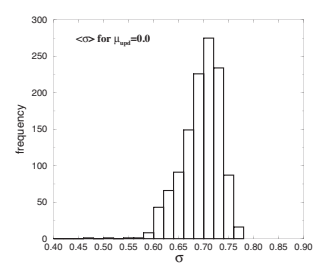

Broken phase

Symm. Phase

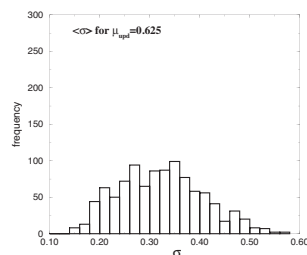

Distributions of the $\langle\sigma\rangle=\langle\bar{\psi} \psi\rangle$ fields

Example of successful reweighting at $\mu \neq 0$ : no conceptual problems 1-dim SU(3) can be exactly solved (Bilic, Demeterfi, 1988) 
Exact Z's zeros in the Imaginary, Real $\mu$ plane (diamonds) and the cloud of zeros obtained from reweighting with a very poor statistics

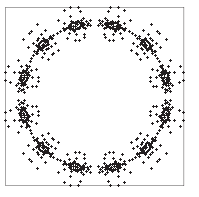

Z's zeros from an high statistics reweighting : OK

Z.Fodor and F.Katz's proposal :

Multiparameter reweighting use fluctuations around $T_{c}$ to explore the critical region

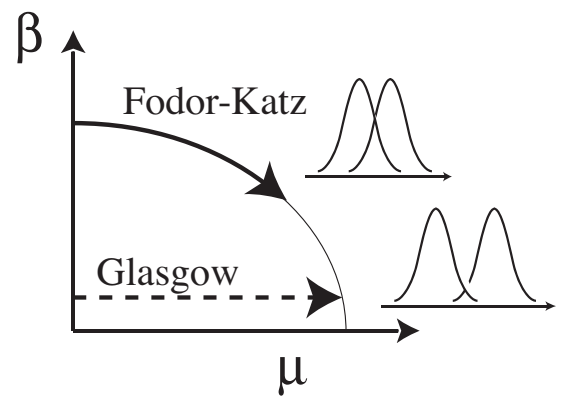




\section{Bielefeld-Swansea}

Taylor expansion of the reweighting factor as a power series in $\lambda=\mu / T$, and similarly for any operator.

Computationally convenient: simplifies calculation of determinant.

Expectation values are then given by

$\langle\mathcal{O}\rangle_{(\beta, \mu)}=\frac{\left\langle\left(\mathcal{O}_{U}+\mathcal{O}_{1} \lambda+\mathcal{O}_{2} \lambda^{2}+\ldots\right) \exp \left(\mathcal{R}_{1} \lambda+\mathcal{R}_{2} \lambda^{2}+\ldots-\Delta S_{g}\right)\right\rangle_{\lambda=U, \beta_{U}}}{\left\langle\exp \left(\mathcal{R}_{1} \lambda+\mathcal{R}_{2} \lambda^{2}+\ldots-\Delta S_{g}\right)\right\rangle_{\lambda=0, \beta_{U}}}$.

Bridge between Canonical and GranCanonical ensembles A. Hasenfratz, D. Toussaint, M. Alford, A. Kapustin, F. Wilczek, ...

$$
\begin{gathered}
\mathcal{Z}(\mu)=\sum_{\mathcal{L}(\mathcal{N}) e^{\left.\beta \mu_{B} N\right)}} \\
\mathcal{Z}(\rangle)=\operatorname{Tre}^{-\beta\left(H-i \nu_{B} N\right)} \\
Z(\mathcal{N})=\frac{\beta}{2 \pi} \int_{0}^{2 \pi / \beta} d \nu \mathcal{Z}(i \nu) e^{-\imath \beta \nu N}
\end{gathered}
$$

Idea: $\mu=0$ fluctuations allow the exploration of $N_{b} \neq 0$ hence tell us about $\mu \neq 0$ Note: same argument suggests Glasgow rewerghtang maght work

Practical Strategy:

$\mathcal{Z}(\mu, T)$ must be

1. analitic

2. non trivial

Rule of thumb:

$\chi(T, \mu)=\partial \rho\left(\mu, T^{\prime}\right) / \partial \mu=\partial^{2} \log Z\left(\mu, T^{\prime}\right) / \partial \mu^{2}>0$ 
$\mu$ Imm.: Lessons from $g=\infty$

$$
\begin{array}{r}
S_{Q C D}=S_{Y M}+S_{F} \rightarrow g \rightarrow \infty=S_{F^{\prime}} \\
\mathcal{Z}=\left(\int V_{\text {eff }}(<\bar{\psi} \psi>d<\bar{\psi} \psi>)^{V_{s}}\right. \\
V_{\text {eff }}(<\bar{\psi} \psi>, \mu)=2 \cosh \left(r N_{t} N_{c} \mu\right)+ \\
\sinh \left[\left(N_{t}+1\right) N_{c}<\bar{\psi} \psi>\right] / \sinh \left(N_{t}<\bar{\psi} \psi>\right) \\
V_{e f f}(<\bar{\psi} \psi>, i \mu)=2 \cos \left(r N_{t} N_{c} \mu\right)+ \\
\sinh \left[\left(N_{t}+1\right) N_{c}<\bar{\psi} \psi>\right] / \sinh \left(N_{t}<\bar{\psi} \psi>\right)
\end{array}
$$
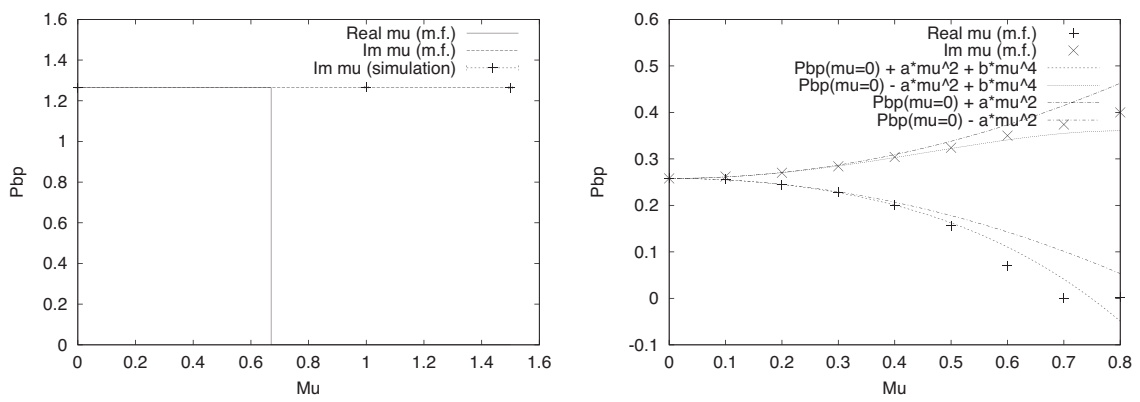

$<\bar{\psi} \psi>$ as a function of real and imaginary $\mu$, for $T \simeq 0$ and $T^{\prime} \simeq T_{c}^{\prime}$ 
Two color QCD as a testbed for Imaginary $\mu_{B}$
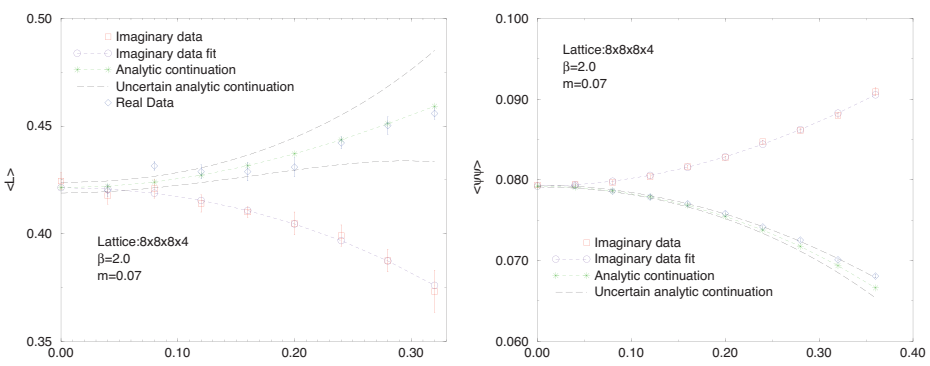

P. Giudice, 'Tesi di L Laurea in Fisica, Universita dẹlla Calabria; Advisor A. Papa

courtesy of the Author

Gross Neveu Mudel

The critical line:

$$
1-\mu / \Sigma_{0}=2 T / \Sigma_{0} \ln \left(1+e^{-\mu / T}\right)
$$

Reduces to:

$$
T\left(T-T_{c}\right)+\mu^{2} /(8 \ln 2)=0
$$

Second order approximation good up to $\mu \simeq T_{c}$ 
EPJ Web of Conferences

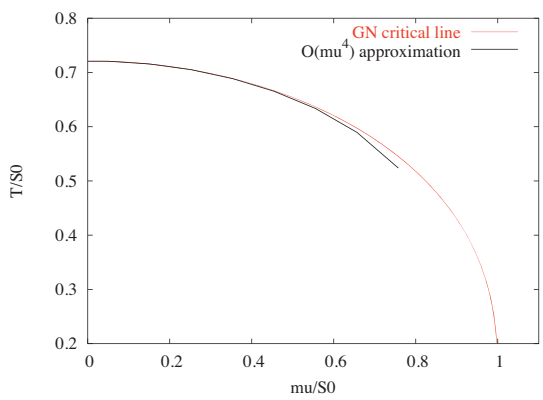

From O. Philipsen and E. Laermann

Ann. Rev. Nucl. Part. Phys. 2003

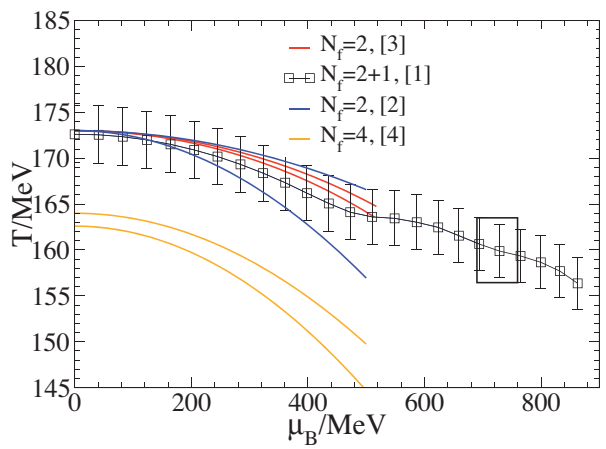

1. Fodor $Z$ and Katz $S D$, JHEP U203:U14 (ZUOZ).

. Altan CR et al., Phys. Rev. D 66:U7ß507 (2uUe).

3. de Forcrand $P$ and Phelapsen O, Nucl. Phys. B642:290 (2002).

4. D'Elra $M$ and Lombardo MP,Phys. Rev. D 1:074507 (2003). 
Lattice QCD at Finite Temperature and Density

Lecture I

I Formulation

II Calculational Schemes

II.1 Effective Fermionic Models - Analytic approaches

II.2 Effective Gluonic Madels - Numerical approaches

III QCD at Finite Baryon Density: Methods

III.1 Derivatives

1II.2 Reweighting

III.3 Expanded Reweighting

III.4 Imaginary Chemical Potential

IV Results - Discussion : Tomorrow's lecture

Helmholtz International Summer School Dense Matter in Heavy Ion Collisions and Astrophysics JINR Dubna Russia July 14-26 2008

QCD at finite temperature and density on the lattice

M.P. Lombardo, INFN Laboratori Nazionali di Frascati 
EPJ Web of Conferences

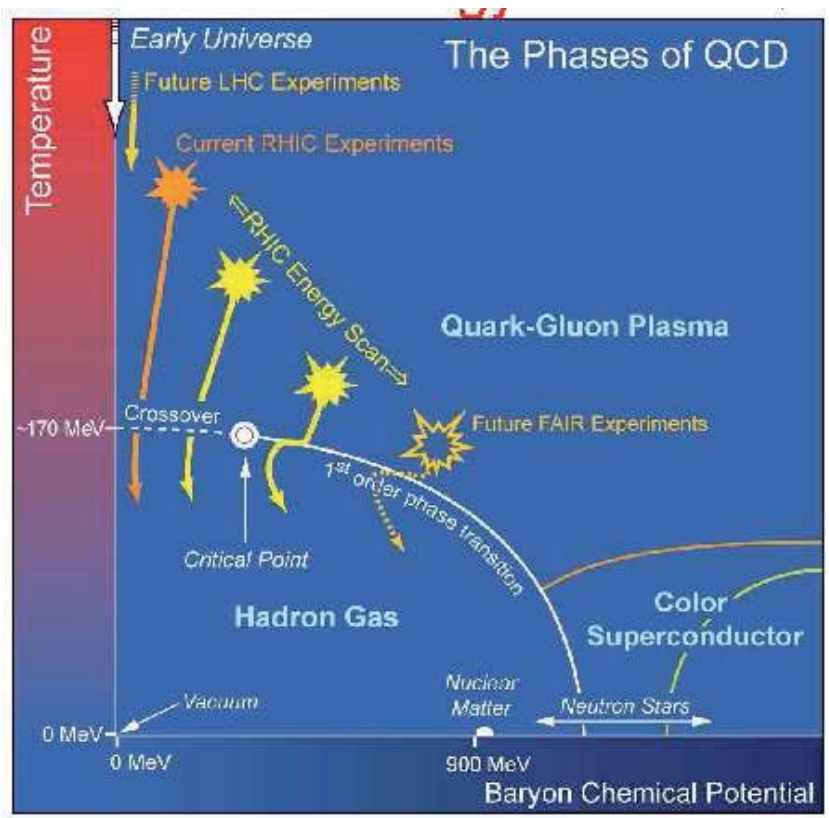

LATTICE FIELD THEORY : FIRST PRINCIPLE APPROACH TO THE QCD PHASE DIAGRAM

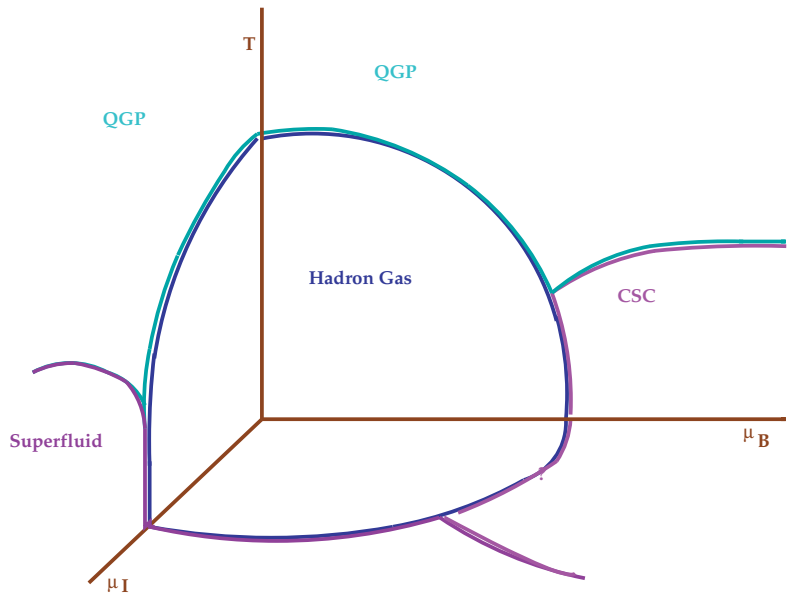



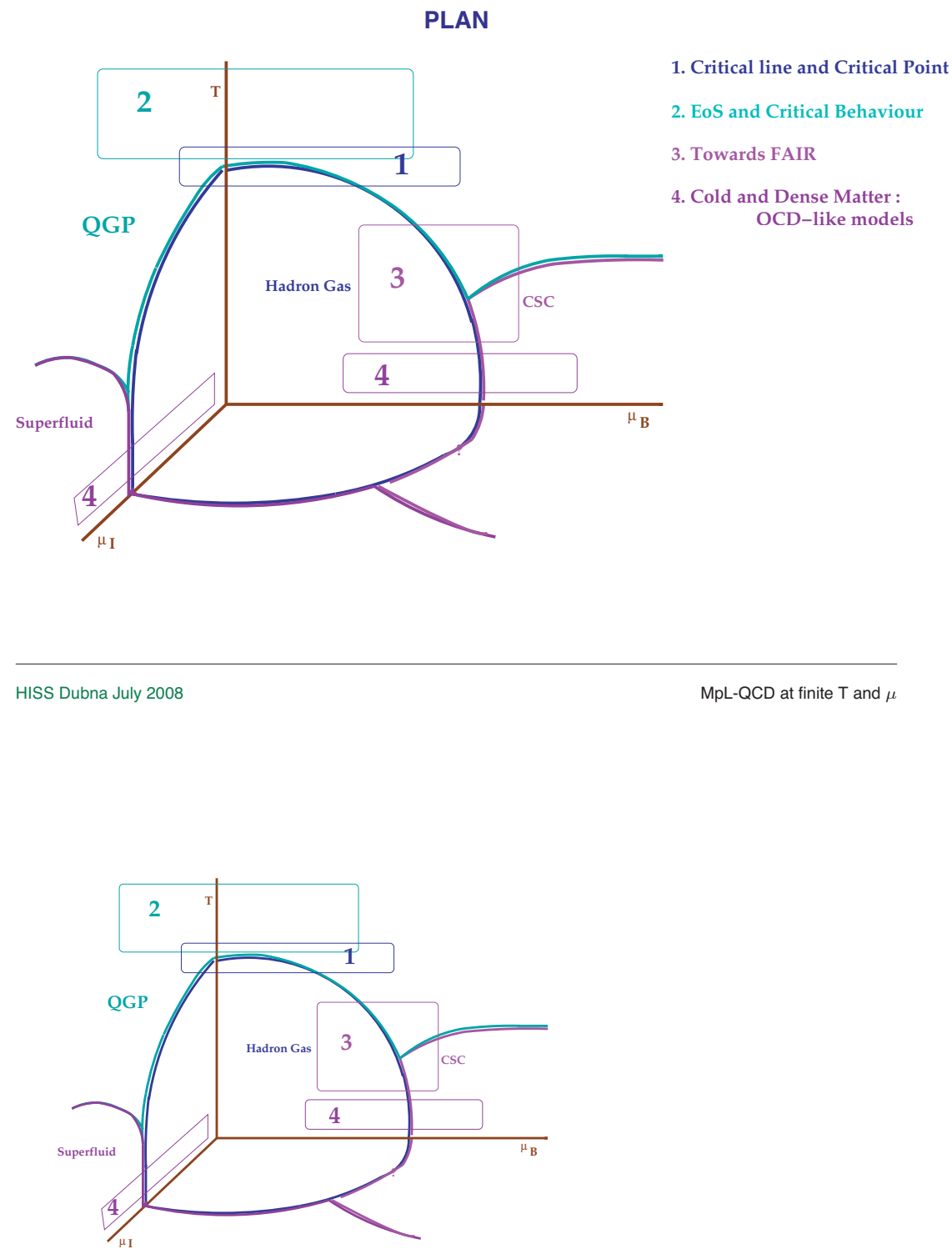

1. $T_{c}$, THE CRITICAL LINE AND THE CRITICAL POINT 
EPJ Web of Conferences

$T_{c}$ :

RBC-Bielefeld

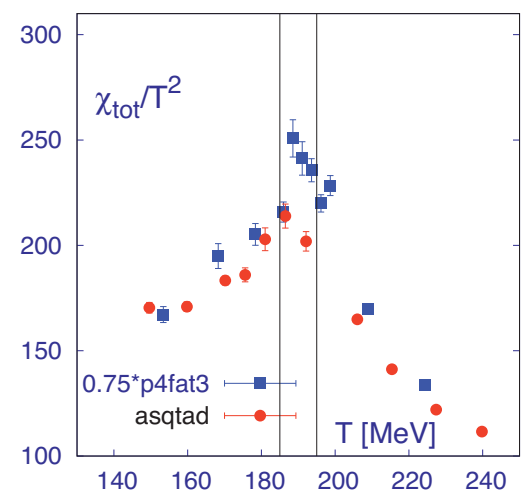

$T_{c}$ :

WUPPERTAL-JUELICH

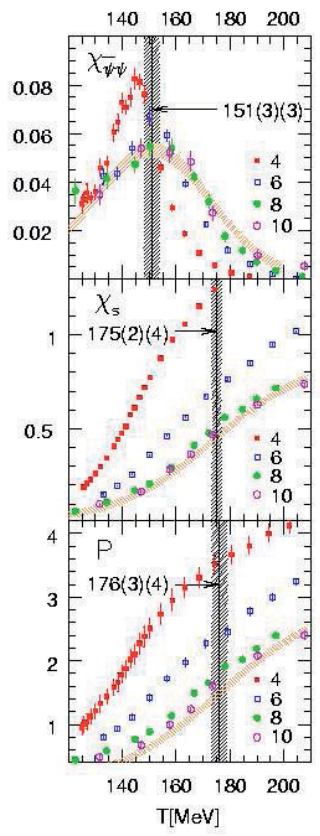


$T_{c}$ AT $\mu_{B}=0:$ STATUS AS OF QM2008

RBC-BIELEFELD :

$T_{c}=190(5) \mathrm{MeV}$

WUPPERTAL-JUELICH :

$T_{c}=175(5) \mathrm{MeV}$ (Glue)

$T_{c}=151(6) \mathrm{MeV}$ (Fermions)

THE CRITICAL

LINE IN THE $\mu_{B}, T$ PLANE AT SMALL $\mu_{B}$

$$
\begin{aligned}
& T=T_{c}-K \mu_{B}^{2} \\
& K \propto 1 / N_{f}
\end{aligned}
$$

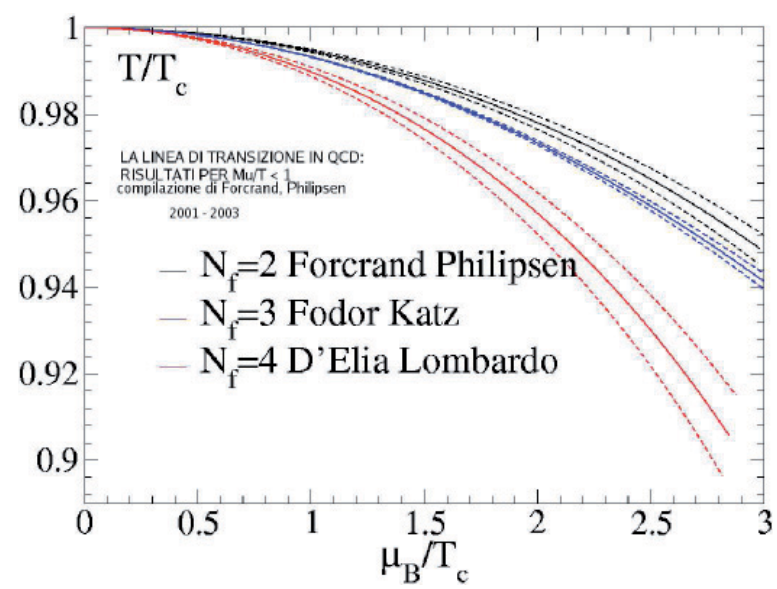


TOWARDS THE CONTINUUM LIMIT:

CURVATURE $K$ OF THE CRITICAL LINE IN THE $\mu_{B}$-T PLANE

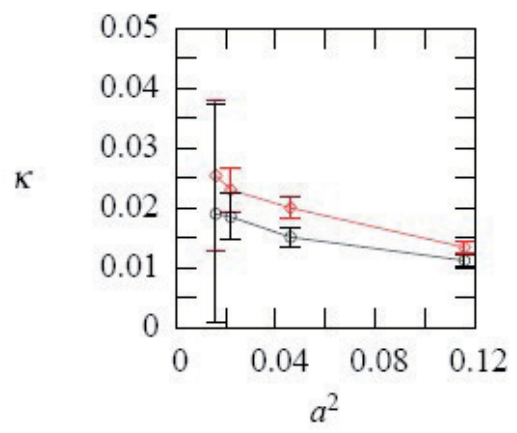

from gluonic and fermionic susceptibilities

Results for $\mathrm{Nf}=2+1$

Fodor, Guse, Katz, Kálmán K. Szabó, QM2008

\section{The Critical EndPoint of QCD}

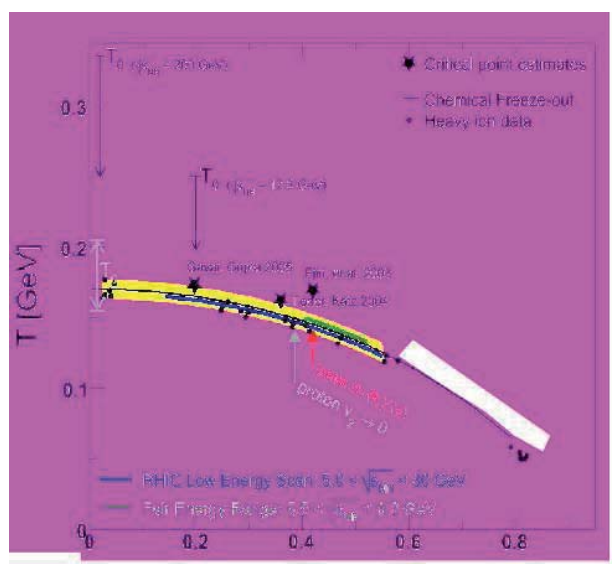




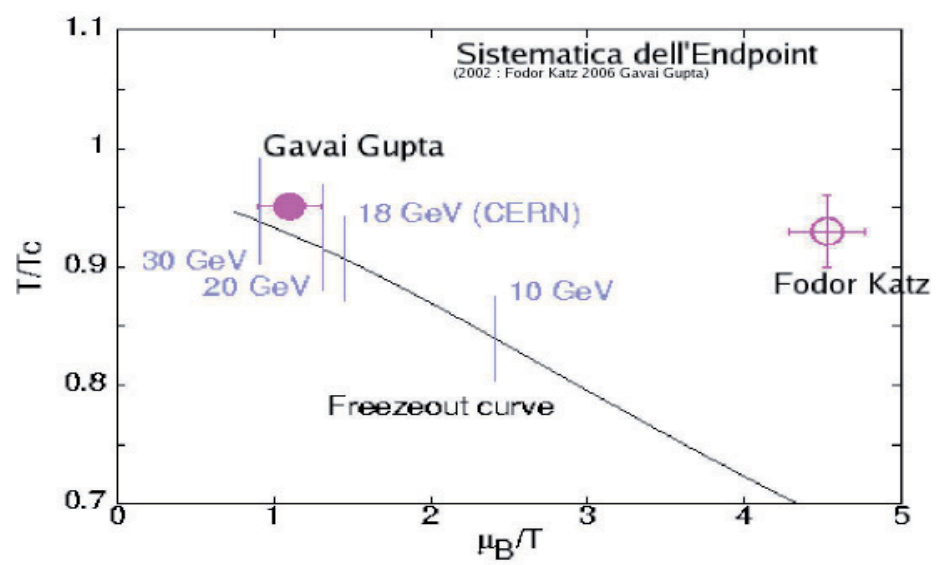

\section{Challenging the CRITICAL EndPOINT OF QCD}

\section{Forcrand-Philipsen : strategy}

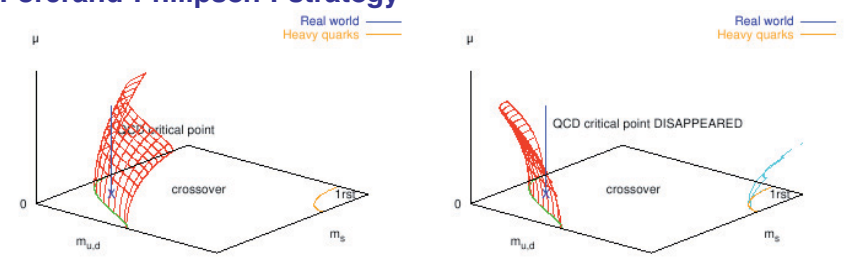

Scenario I or Scenario II ? To decide, measure slope $K$ in

$$
\frac{m_{c}(\mu)}{m_{c}(0)}=1+K\left(\frac{\mu}{T}\right)^{2}+\ldots
$$

$K>0:$ Scenario I, critical endpoint at small $\mu_{B}$

$K<0$ : Scenario II, NO critical endpoint at small $\mu_{B}$ : favored at QM2006 
Forcrand-Philipsen Lat07 : improve and confirm previous results

$$
\frac{m_{c}(\mu)}{m_{c}(0)}=1-3.3(5)\left(\frac{\mu}{T}\right)^{2}+\ldots
$$

Kogut-Sinclair 2007 : QCD at finite isospin density NB : $\mu_{I} \simeq \mu_{B}$ at $T \simeq T_{c}$ Toublan, Kogut, Sinclair 2004

$$
\frac{m_{c}(\mu)}{m_{c}(0)}=1-3 .(1)\left(\frac{\mu_{I}}{T}\right)^{2}+\ldots
$$

\section{Current Results:}

- Confirm unusual scenario for $N_{t}=4$

- Suggest NO critical endpoint for $\mu_{B}<600 M e V$

- See talk by Philippe de Forcrand

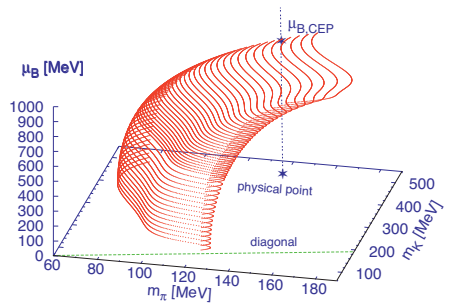

Kovács and Szép, 2006 compute critical surface of the $S U(3)_{L} \times S U(3)_{R}$ chiral quark model at non-zero baryon density.

Conventional Scenario realized in NJL, $\mu_{B}^{C E P} \sim 900 \mathrm{MeV}$.

Qualitative difference between QCD and NJL at small $\mu_{B}:$ : Details of the dynamics important. 


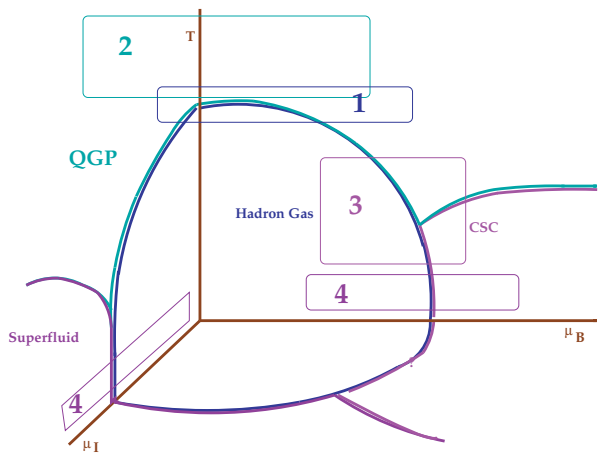

\section{EOS AND CRITICAL BEHAVIOUR}

\section{LATTICE OBSERVABLES FOR THERMODYNAMICS}

Number Density : accessible at imaginary chemical potential.

$$
n_{u, d}\left(T, \mu_{u}, \mu_{d}, m_{u}, m_{d}\right)=\frac{\partial p\left(T, \mu_{u}, \mu_{d}\right)}{\partial \mu_{u, d}} ; p\left(T, \mu_{u}, \mu_{d}\right)=\frac{T}{V} \ln Z\left(T, \mu_{u}, \mu_{d}\right)
$$

Susceptibilities: accessible at at $\mu=0$

$$
\chi_{j u, j_{d}}(T)=\left.\frac{\partial^{\left(j u+j_{d}\right)} p\left(T, \mu_{u}, \mu_{d}\right)}{\partial \mu_{u}^{j u} \partial \mu_{d}^{j_{d}}}\right|_{\mu_{u}=\mu_{d}=0}
$$

Test for fluctuations.

Taylor coefficients of the excess pressure:

$\Delta p\left(T, \mu_{u}, \mu_{d}\right) \equiv p\left(T, \mu_{u}, \mu_{d}\right)-p\left(T, \mu_{u}=0, \mu_{d}=0\right)=\sum_{j_{u}, j_{d}} \chi_{j_{u}, j_{d}}(T) \frac{\mu_{u}^{j_{u}}}{j_{u} !} \frac{\mu_{d}^{j_{d}}}{j_{d} !}$,

containing information about baryon density effects in the EoS. 


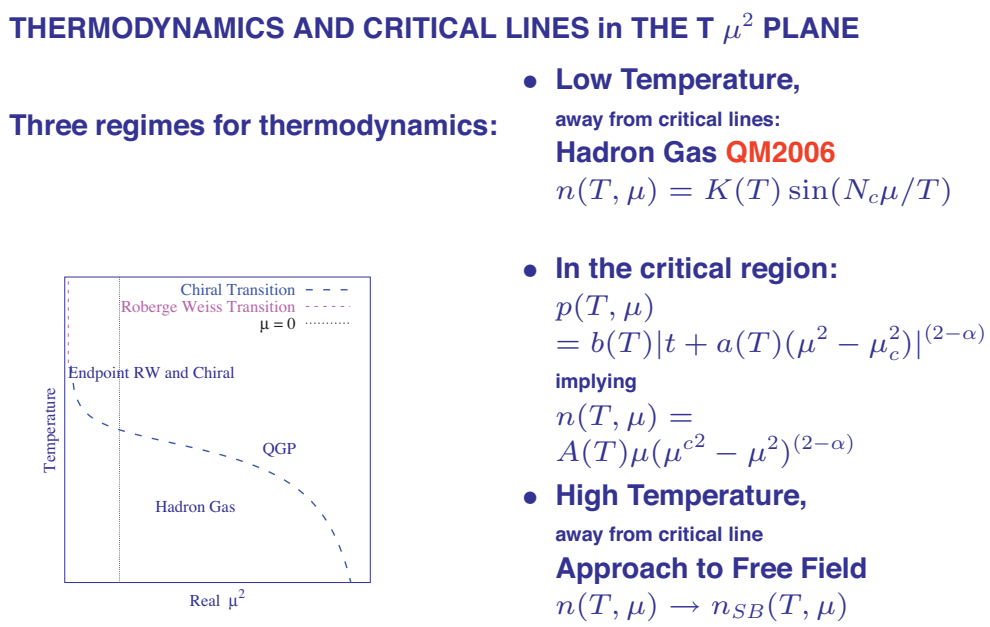

HADRON GAS; CRITICAL BEHAVIOUR; SB from SUSCEPTIBILITIES
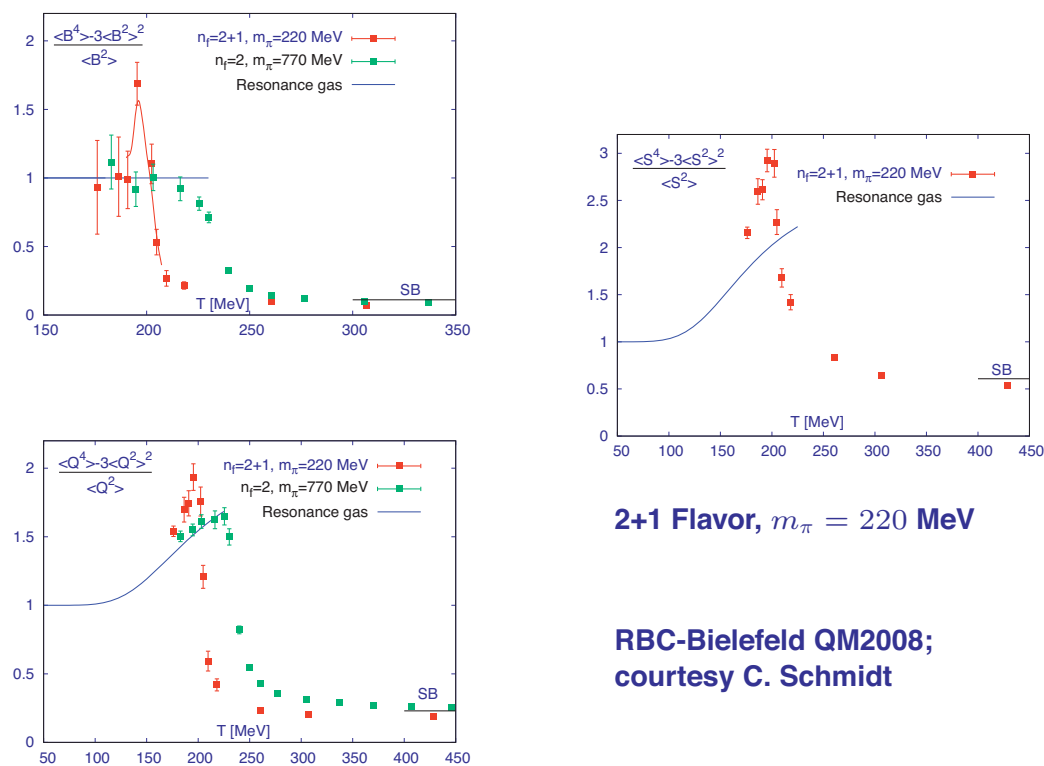

2+1 Flavor, $m_{\pi}=220 \mathrm{MeV}$

RBC-Bielefeld QM2008; courtesy C. Schmidt 


\section{CRITICAL BEHAVIOR AND THERMODYNAMICS}

\section{AT THE ENDPOINT OF THE RW TRANSITION}

Critical behavior at imaginary $\mu$

Continued to real $\mu$.

$n\left(\mu_{I}\right)=A(T) \mu_{I}\left(\mu^{c^{2}}-\mu_{I}^{2}\right)^{(2-\alpha)}$

$$
\begin{aligned}
& n(\mu)=A(T) \mu\left(\mu^{c 2}+\mu^{2}\right)^{(2-\alpha)} \\
& \left.n_{S B}(\mu)=A \mu+B \mu^{3}\right) \rightarrow \alpha=1
\end{aligned}
$$
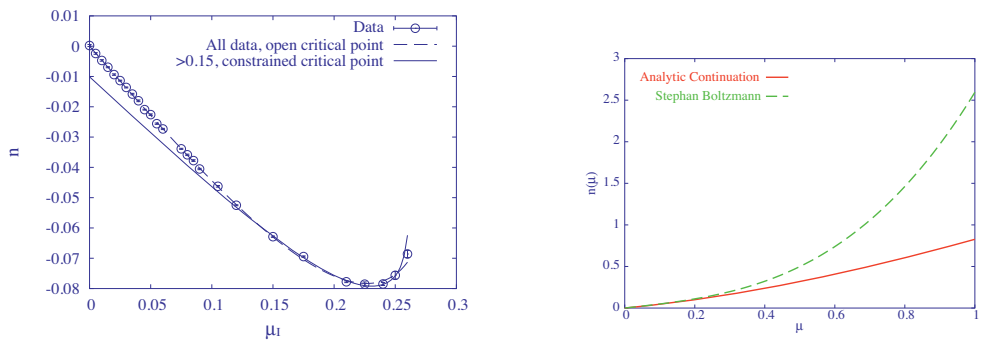

D’Elia, Di Renzo, Lombardo, 2007, QM2008

CRITICAL BEHAVIOR, THERMODYNAMICS, QUASIPARTICLE MODELS

Kämpfer, Bluhm Proposal

amenable to an easy comparison with lattice data.

I. Quasiparticlemodel vs Imaginary Chemical Potential Lattice Data, and analytic continuation to Real Chemical Potential
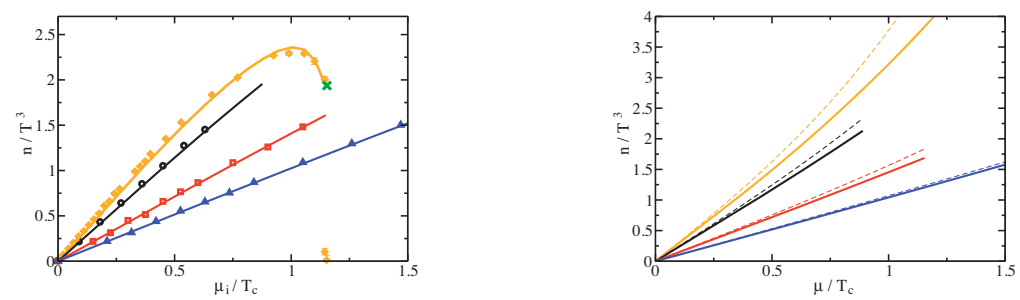

Kämpfer, Bluhm , 2007, QM2008 
EPJ Web of Conferences

II. Quasiparticlemodel vs Taylor Coefficients
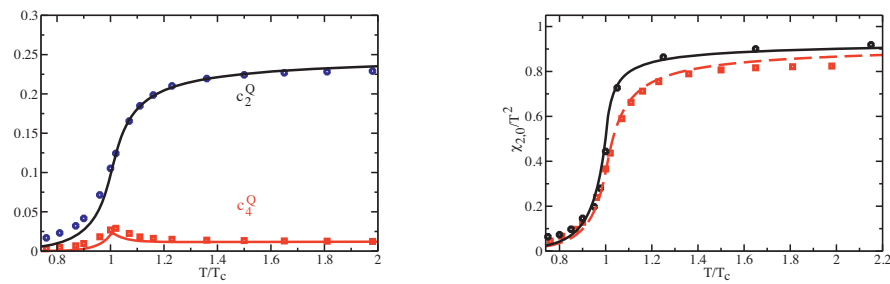

Crucial ingredients:

- Explicit dependence of the self-energy parts on $\mu_{i}=\mu_{u, d}$ and $T$

- Implicit dependence via the effective coupling $G^{2}\left(T, \mu_{u}, \mu_{d}\right)$.

$$
\omega_{i}^{2}=k^{2}+m_{i}^{2}+\Pi_{i}, \quad \Pi_{i}=\frac{1}{3}\left(T^{2}+\frac{\mu_{i}^{2}}{\pi^{2}}\right) G^{2}\left(T, \mu_{u}, \mu_{d}\right) .
$$

Kämpfer-Bluhm : see Poster

\section{APPROACH TO SB : ANALYTIC RESULTS VS. LATTICE DATA}

\section{Based on A. Vuorinen, 2004}
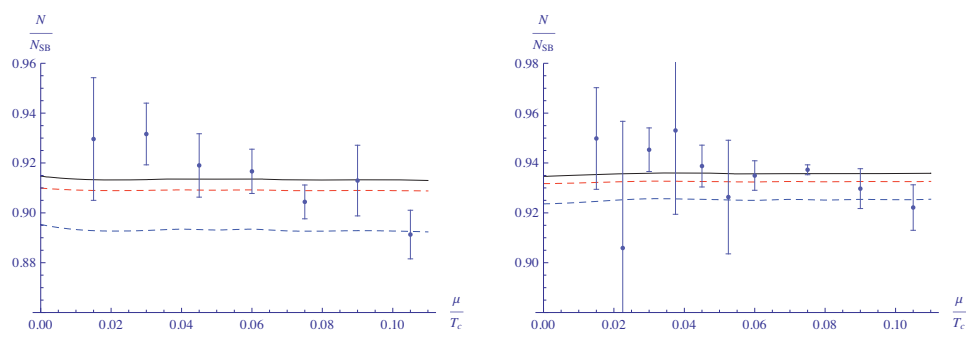

$T=1.5 T_{c}$

$$
T=3.5 T_{c}
$$

D'Elia, Di Renzo, Lombardo, Vuorinen, in progress

Lattice Cutoff Effects : Karsch, Laermann, 2008 


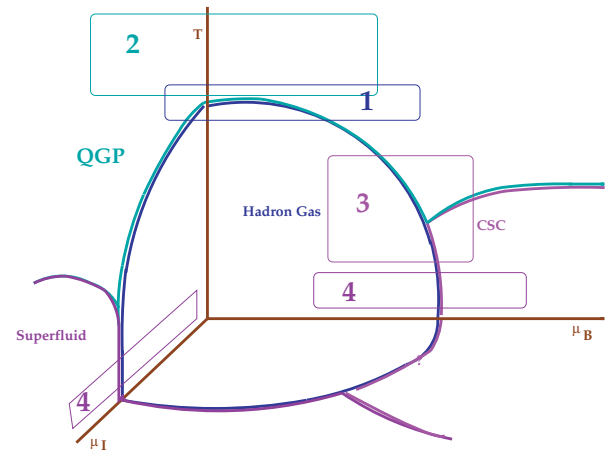

\section{TOWARDS FAIR}

\section{STRONG COUPLING}

New Results on The Mass Spectrum
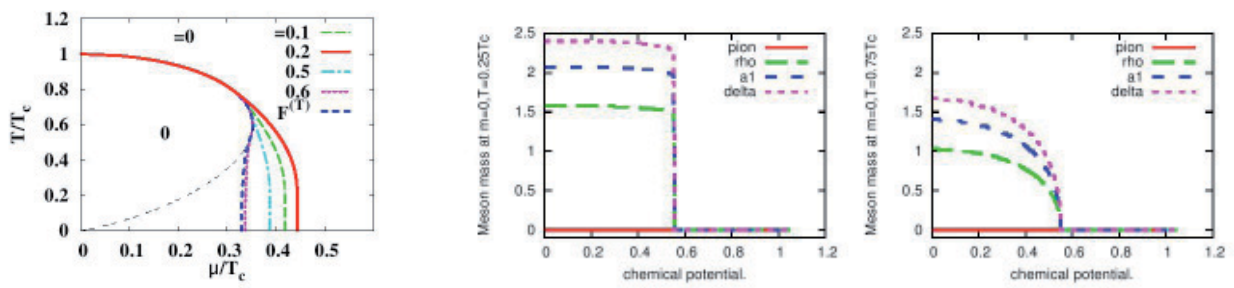

Kawamoto, Miura 2007
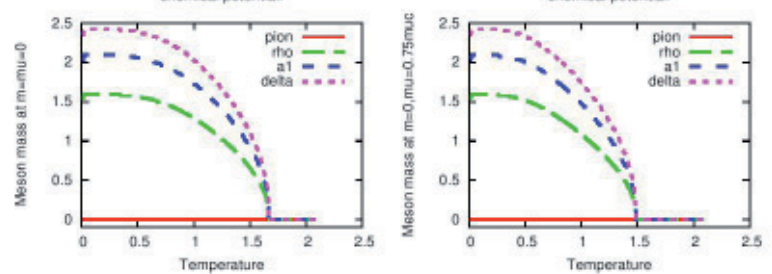
HEAVY QUARK EFFECTIVE MODEL

Double limit: $M \rightarrow \infty, \mu \rightarrow \infty, \zeta \equiv \exp (\mu-\ln M):$ Fixed Evolved 'quenched approximation' in the presence of charged matter

\section{Polyakov Loop}

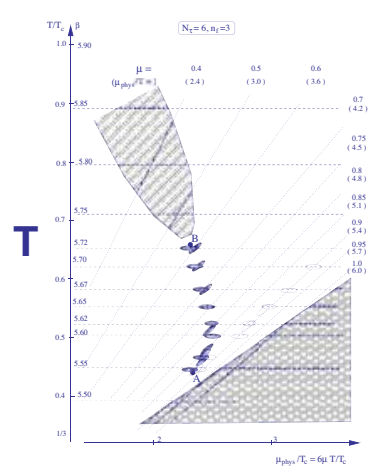

$\mu_{B}$

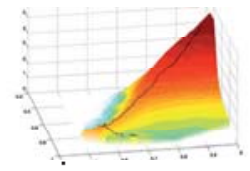

Results for $N_{f}=3$ :

- Identified phase transition

- Indentified Ridge in the T, $\mu$ plane

- Studies of diquark in progress

Di Pietro, Feo, Seiler, Stamatescu 2008

\section{CANONICAL FORMALISM}

$\mathcal{Z}_{\mathrm{C}}(T, N)=$

$\frac{3}{2 \pi} \int_{0}^{2 \pi T} d\left(\mu_{I} / T\right) e^{-N i \mu_{I} / T} \mathcal{Z}_{\mathrm{GC}}\left(T, i \mu_{I}\right)$

Forcrand and Kratovchila, 2006,2007

Canonical partition a la Hasenfratz-Toussaint:

$\frac{Z_{C}(B, \beta)}{Z_{G C}\left(\beta_{0}=\beta, \mu=i \mu_{0}\right)}$

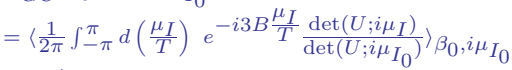

$\equiv\left\langle\frac{\hat{z}_{C}(U ; B)}{\operatorname{det}\left(U ; i \mu_{0}\right)}\right\rangle_{\beta_{0}, i \mu_{I_{0}}}$

Results for $N_{f}=4$ :

- First order line-coexistence region

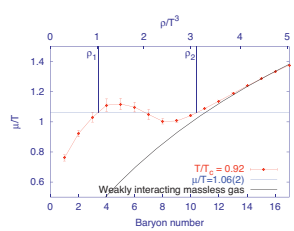

- Critical $\mu$ and Critical densities $\rightarrow$

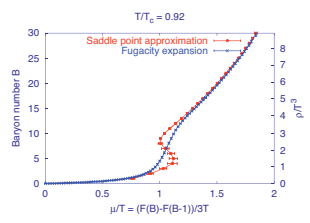


CANONICAL APPROACH II : HISTOGRAMS

Proposed by Ejiri,2007

Alternative search for a critical point in $N_{f}=2 \mathrm{QCD}$, large masses

Alexandru, Li, Liu, 2007 :

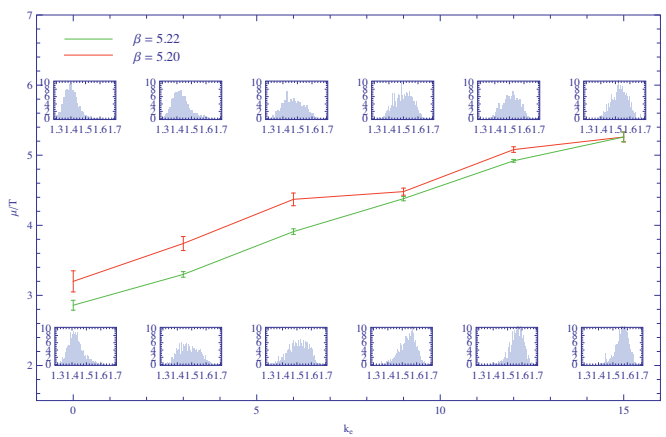

\section{CANONICAL APPROACH III :}

Grand Partition Function via a Taylor Expansion.

Ejiri, 2007,2008

$$
\begin{aligned}
& \frac{\mathcal{Z}_{\mathrm{GC}}\left(T, \mu_{q}\right)}{\mathcal{Z}_{\mathrm{GC}}(T, 0)} \equiv\left\langle e^{\left[N_{\mathrm{f}} N_{t} V \sum_{n=1}^{\infty} D n\left(\frac{\mu_{q}}{T}\right)^{n}\right]}\right\rangle_{\left(T, \mu_{q}=0\right),} \\
& \frac{\mathcal{Z}_{\mathrm{C}}(T, \bar{\rho} V)}{\mathcal{Z}_{\mathrm{GC}}(T, 0)} \text { at large } \mathbf{V} \approx\left\langle\exp \left[V\left(N_{\mathrm{f}} N_{t} \sum_{n=1}^{\infty} D_{n} z_{0}^{n}-\bar{\rho} z_{0}\right)\right] e^{-i \alpha / 2} \sqrt{\frac{1}{V\left|D^{\prime \prime}\left(z_{0}\right)\right|}}\right\rangle_{(T, \mu=0)}
\end{aligned}
$$

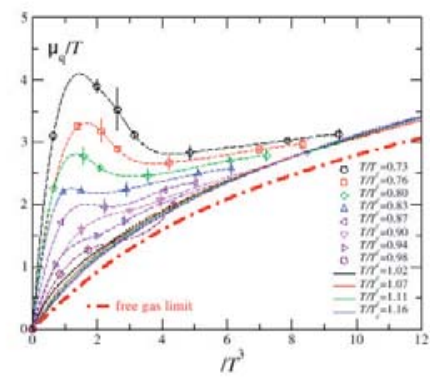

Results for $N_{f}=2$ :

- Qualitative change at $T / T_{c} \simeq 0.8$

- Indication of First Order Line

Ejiri 2008 
DENSITY OF STATES

Luo, Azcoiti et al,, Ambjorn et al., Anagnostopoulos and J. Nishimura, $<O\rangle=\int d \phi\langle O f(U)\rangle_{\phi} \rho(\phi) / \int d \phi\langle f(U)\rangle_{\phi} \rho(\phi), \phi$ fixed

Density of states $-\rho-$ constrained partition function: $\rho(x)=\int \mathcal{D} U g(U) \delta(\phi-x)$.

Results for $N_{f}=4$

- Signal of two phase transition lines

- Indication for a triple point

Fodor, Katz, Schmidt 2007
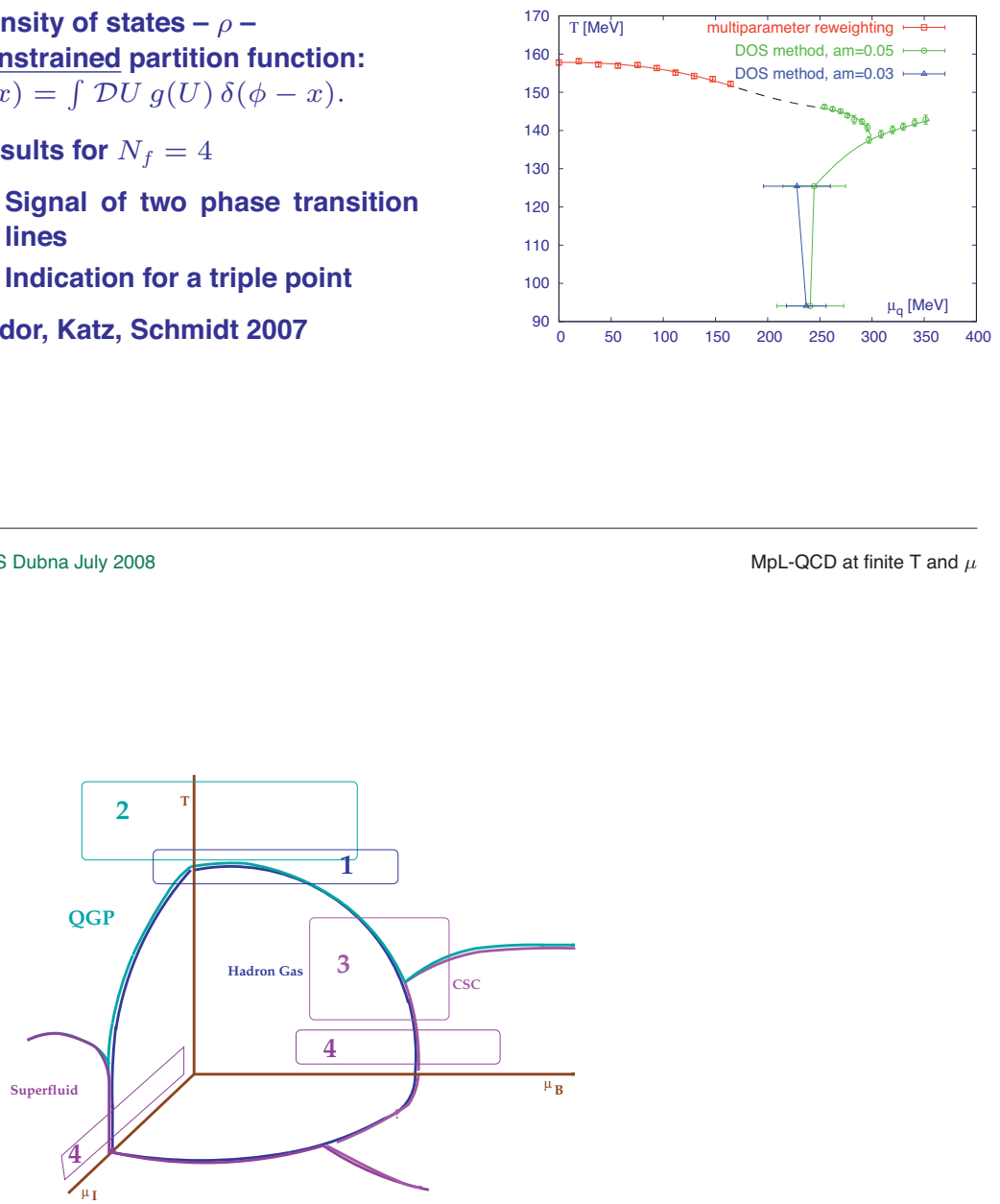

4. COLD AND DENSE MATTER 


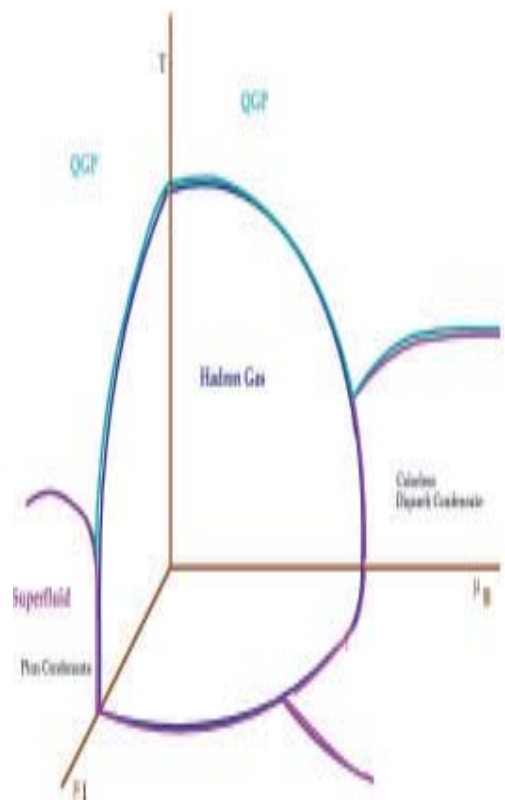

\section{TWO COLOR QCD}

- Symmetry : $q q \longleftrightarrow \longrightarrow \bar{q} q$

- Symmetry : $\mu_{B} \leftarrow \rightarrow \mu_{I}$

- At T=0 $\mu_{B}^{c}=\mu_{I}^{c}=m_{\pi} / 2$

- Diquark Condensate for $\mu_{B}>\mu_{B}^{c}$

- Pion Condensate for $\mu_{B}>\mu_{I}^{c}$

- No sign problem

QCD at $\mu_{I} \neq 0$ and TWO COLOR QCD at $\mu_{B} \neq 0$

\section{No sign problem, many studies!}

- Low Temperature, Fermionic Sector

- Strong coupling analysis [Dagotto, Karsch, Moreo,Wollf, 1986]

- Laboratory for diquark condensation [Alford,Rajagopal and Wilczek; Rapp, Schäfer, Shuryak and Velkovsky, 1998]

- Numerical studies of symmetries and spectrum [Hands, Kogut, Morrison, MpL 1998]

- Lattice studies of diquark condensation [Hands, Kogut, Morrison, Sinclair;1998; Aloisio, Azcoiti, di Carlo, Galante, 1999]

- RMT analysis [Akemann, Splittorff, Toublan, Verbaarschot,2001]

- Studies of the Dirac spectrum [Bittner, Markum, Pullirsch, MpL, 2001]

- $\mu_{C}(T=0)=m_{\pi} / 2:$ amenable to $\chi \mathbf{P T}$ analysis.

[Kogut, Stephanov, Toublan, Verbaarschot and Zhitnitsky, 2001; Kogut, Toublan, Sinclair 2002]

- EoS and Gluon Condensate from $\chi$ PT [Metlitsky,Toublan,Zhitnisky, 2005,2006]

- Model studies of the vector sector [Lenaghan, Sannino, Splittorff 2002]

- Vector spectroscopy on the lattice [Alles, D'Elia, Pepe, MpL, 2001; Muroya,Nakamura,Nonaka, 2003]

- Test Bed for Imaginary $\mu$ [Cea, Cosmai, Giudice, D'Elia, Papa, 2006, 2007]

- High T, Gluon Sector finite $\mu$ transition from hadron gas to QGP, similar to the $\mathrm{T}=0$ transition

- Critical Line [Kogut, Sinclair, 2003-2008]

- Topological Susceptibility [ Alles, D'Elia, Pepe, MpL 2002]

- Polyakov Loop [Muroya,Nakamura, 2004; Alles, D'Elia, MpL 2006]

- Order Parameter for Cofinement [D'Elia Conradi 2007]

- Critical Line [Forcrand, Stephanov, Wenger, 2007]

- Low T : Chiral Symmetry, Confinement, BEC Phase

- Confinement/Deconfinenement,Screening [Hands, Kim, Skullerud 2006-2008]

- Glueballs spectrum, screening [Lombardo, Paciello, Petrarca, Taglienti, 2007]

- BEC Phase, interface BEC/QGP [Forcrand, Stephanov, Wenger, 2007] 
THE PHASE DIAGRAM FOR A FINITE ISOSPIN DENSITY

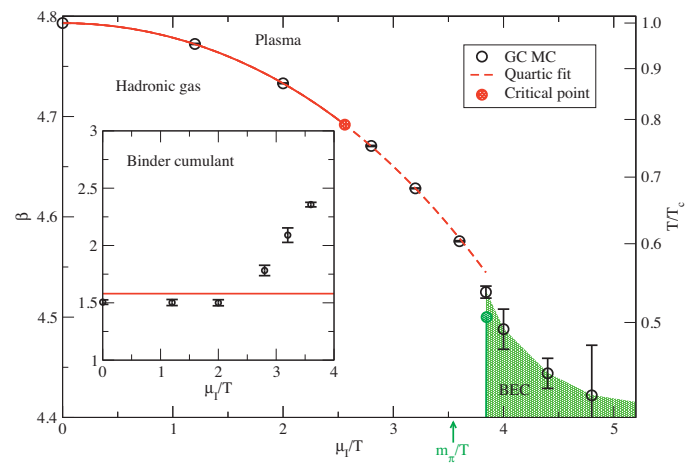

Forcrand, Stephanov, Wenger, 2007

\section{GLUONIC OBSERVABLES IN THE BEC PHASE of $\mathbf{Q C}_{2} \mathbf{D}$}

$0^{++}$Glueball : lighter in the BEC phase

Susceptibility: $\chi=<P^{2}>-<P>^{2}$ peaks at $\mu_{c}$

\begin{tabular}{cc}
\hline $\begin{array}{c}\text { Normal Phase } \\
m_{\pi} / m_{\rho}\end{array}$ & $m_{0}^{++} / m_{\rho}$ \\
\hline 0.40 & 1.07 \\
0.42 & 1.26 \\
\hline BEC & \\
\hline 0.64 & 0.80 \\
0.80 & 0.23
\end{tabular}

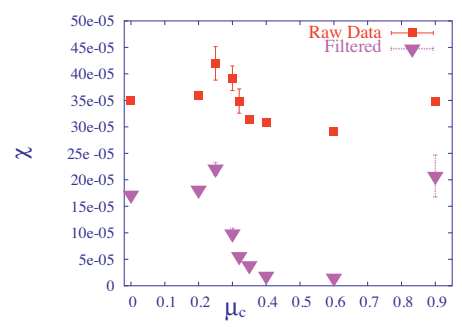

Lombardo,Petrarca,Paciello,Taglienti,2007 
BEC AND CONFINEMENT: deconfinement deep in the BEC phase

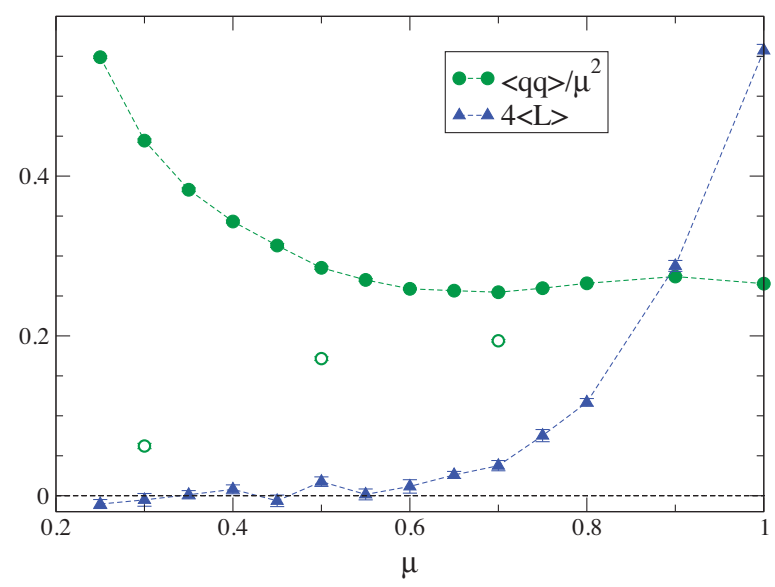

Hands, Kim, Skullerud 2006,2007
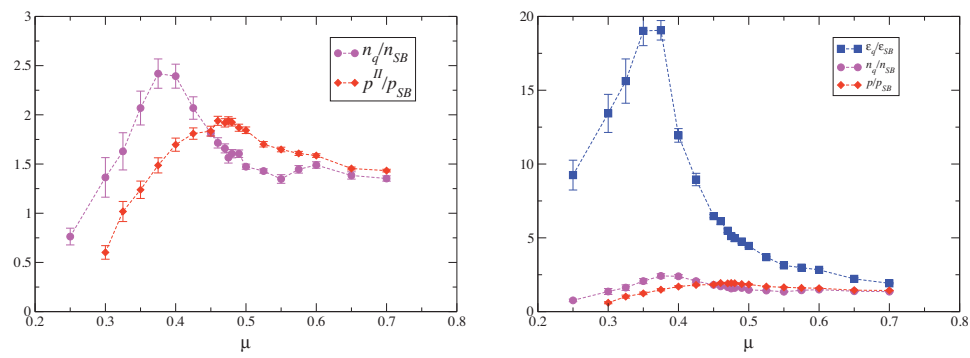

Hands, Kim, Skullerud 2008; preliminary, courtesy S. Hands 


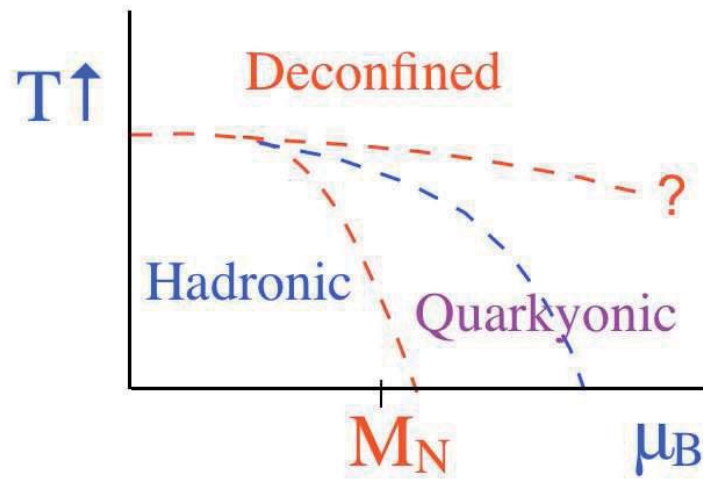

From Small $N_{c}=2$ to

Large $N_{c}$ : Proposed Phase Diagram

McLerran, Pisarski, 2007

QCD AT FINITE TEMPERATURE AND DENSITY : SUMMARY

$T_{c}$ known within $20 \%$.

Fate of the critical endpoint of QCD???

Small $\mu$ : Mature calculations. Critical line towards continuum Mass spectrum depends on $\mu_{B}$

Critical line at lower temperature, larger $\mu$ 'bends down' First evidences of a triple point in the $\mathbf{T}, \mu_{B}$ plane

Cold Phases studied at $\mu_{I} \neq 0 / \mathbf{Q} C_{2} \mathbf{D}$ at $\mu_{B} \neq 0$

Examples of interesting patterns confinement/chiral symmetry.

Critical region needs exact dynamics, models do not suffice Qualitative difference critical surface QCD / NJL, HRG fails around $T_{c}$ 